\title{
Results and perspective
}

\author{
Nadezhda N. Efremova
}

The long large-scale work on monitoring the state of monuments in St. Petersburg, the results whereof are presented in this collective monograph, and which pooled the efforts of the museum staff and St. Petersburg scientists, has the ultimate goal of putting into action a scientific strategy for the preservation of the monuments of cultural heritage.

Integrated monitoring provides an objective picture of the state of the materials of monuments in the urban environment, makes it possible to take timely interventions for the restoration and conservation of works of art, to plan the necessary work.

Proceeding from the conducted scientific research, a description of the rare in its variety and beauty collection of natural stone in the museum Necropoleis, of the types and processes of deterioration of the materials of St. Petersburg monuments under the influence of physicochemical and biogenic factors was made. The unique material obtained on the processes of destruction of monuments in St. Petersburg allows to develop scientific strategies for preservation of monuments in other cities with similar climatic and ecological conditions.

Many years of fruitful experience in monitoring and preserving the unique collection of monumental art makes it possible to implement a major international project. It is there that a global perspective is seen in the cooperation of museum professionals and scientists.

Of course, further streamlining of the monitoring methods will be necessary, taking into account the continuous development of computer technologies, the new achievements in various fields of natural science, enabling us to study in detail the specifics of the interaction of the material of the monumental sculpture with the environment.
Studies should be continued, aimed at searching for effective protection of monuments made from various materials (stone, bronze, cast iron, etc.). A comprehensive study of the properties and causes of deterioration of various types of stone and metal alloys is the key to the successful selection of restoration methods and tools from the wide range of materials offered by the modern science. Therefore, preliminary examinations, scientific research of monument materials and the products of their deterioration should be mandatory when developing the restoration techniques. It is the monitoring system that allows you to objectively determine the need, timing and scope of the next complex restoration.

A particular attention should be paid to monitoring the condition of monuments after restoration and conservation work, to be largely determined by the properties of the materials and methods used. It is essential that the specific aspects of the monument's structure were taken into account, including all internal supporting elements. To this end, it is necessary to use more actively modern research tools that allow quantification of the occurring changes (ultrasonic sounding, laser scanning, etc.).

For any museum, restoration is a special kind of custodial work, for which the research component is fundamental. That is why it is hard to overestimate the need for further scientific research aimed at performance-enhancing methods of restoration and conservation.

The study of the impact of the environment on the state of the monument materials, the search for effective ways to protect them, make it possible to preserve and adequately exhibit the works of monumental sculpture and memorial art, which are an impressive, imaginative part of the world history and culture.

N. N. Efremova $(\square)$

State Museum of Urban Sculpture, St. Petersburg, Russia

e-mail: monument.mgs@gmail.com 